\title{
Sociodemographic Characteristics of Acne among University Students in Damascus, Syria
}

\author{
Waqar Al-Kubaisy, ${ }^{1}$ Nik Nairan Abdullah, ${ }^{1}$ Sabzali Musa Kahn, ${ }^{2}$ and Maram Zia ${ }^{3}$ \\ ${ }^{1}$ Population Health \& Preventive Medicine, Faculty of Medicine, Universiti Teknologi MARA (UiTM), \\ Sungai Buloh Campus, Jalan Hospital, 47000 Sungai Buloh, Selangor, Malaysia \\ ${ }^{2}$ Cultural Centre, University of Malaya, Lembah Pantai, 50603 Kuala Lumpur, Malaysia \\ ${ }^{3}$ Faculty of Medicine, Syrian International University for Science and Technology (SIUST), Dara'a, Damascus, Syria
}

Correspondence should be addressed to Nik Nairan Abdullah; niknairan@yahoo.com

Received 15 April 2013; Revised 7 November 2013; Accepted 20 November 2013; Published 2 January 2014

Academic Editor: Suminori Kono

Copyright (C) 2014 Waqar Al-Kubaisy et al. This is an open access article distributed under the Creative Commons Attribution License, which permits unrestricted use, distribution, and reproduction in any medium, provided the original work is properly cited.

To estimate the prevalence and identify risk factors that may be associated with acne among university students in Syria, a crosssectional study was conducted in the Syrian International University for Science and Technology in December 2009. A sample of 500 students was chosen. Each participant was subjected to an interview and clinical examination of acne in addition to height and weight measurements. Acne prevalence was 34.7\% (172/496). Male students had higher rate of acne compared to females (42.9\% versus $23.6 \%, P<0.0001)$ and their acne started significantly at a younger age (18.13 versus 19.04 years old, $P<0.0001)$. Face was the commonest site for acne in both males and females. Washing face frequently per day in both sexes has a significant relation with a decreased prevalence of acne. Moreover, psychological stress particularly when the students were away from family was associated with a significant higher rate of acne. We found that the prevalence of acne steadily increased with increasing body mass index. Acne is a health and psychological problem among university students particularly when affecting the face. Several factors such as gender, body mass index, and stress were found to be associated with acne formation.

\section{Introduction}

Vulgaris is a common skin disease which is a significant health problem among adolescents and young adults. It affects $85-100 \%$ of people at some point in their lives, and it usually begins at puberty. Acne can persist into the 30s and beyond. People of all ethnic backgrounds get acne [1] More than half of those who experienced acne sought medical treatment. In USA, $61.9 \%$ of patients aged 18 years and older were seen in clinics for acne vulgaris [2]. Likewise in the university outpatient clinics, the most common complaint was acne vulgaris [3].

Acne formation is multifactorial. The four primary factors determining acne development are abnormal follicular epithelial desquamation, hyperactivity of the sebaceous glands, proliferation of Propionibacterium acnes, and follicular formation [4]. Furthermore, androgens play an important role in the pathogenesis of acne. Several studies in the Middle East reported that emotional stress, premenstrual factors (in females), and certain foods such as fatty food, sweets, and spices were believed to aggravate acne formation $[5,6]$. On the other hand, in Syria, there is a lack of published studies on acne among university students. Studying acne is important because some forms of acne such as inflammatory acne may leave scarring and psychological consequences. Presence of acne has a negative impact on self-esteem and quality of life among adolescents. Therefore, increasing incidence of depression and other psychological disorders may be associated with acne [7]. Our objective of conducting this study was to estimate the prevalence of acne among the students in the Syrian International University for Science and Technology and also to identify some risk factors that may be associated with acne.

\section{Methods}

A cross-sectional study was conducted in Dara'a district of Damascus, Syria, from the 3rd to 28th of December 2009. The 
study was carried out on 2100 students of the Syrian International University for Science and Technology (SIUST). This new establishment (four years old) consists of six faculties: Medicine, Dentistry, Pharmacy, Petroleum Engineering, Business Administration, and Computer Science. A stratified cluster sampling method of three stages was implemented for the sample collection. In the first stage, students from each faculty were stratified according to their year level (first, second, third, and fourth) of study. Proportional to the number of students in each level, certain numbers of individuals were randomly chosen. In the second stage the individuals were stratified again and collected proportionally according to their sex. In the final stage, the chosen students from all faculties were grouped into clusters of manageable size.

A random sample of 500 students was chosen. The selected individuals were informed about the purpose of the study and assured about the confidentiality of the information gathered. Interviewing each participant was carried-out using a well-constructed questionnaire. The questionnaire included demographic (age, sex) and social information (living with or away from family) and life style such as washing body times per week (1-2, 3-5, and $>5$ times) and washing face times per day (1-2, 3-5 and $>5$ times) and information about stress frequency (absence, always, and occasional) was also included. In addition, female students were further asked whether acne formation was related to menstrual period. Moreover, clinical examination was carried out by trained two medical doctors who had experience in dermatology. They examined students for the presence and location of acne (such as on the face, shoulder, and others). The clinical examinations were performed at the same time and findings were confirmed between them. Beside that, height and body weight measurements were also taken. Body mass index was categorized into $<20 \mathrm{~kg} / \mathrm{m}^{2}, 20-24 \mathrm{~kg} / \mathrm{m}^{2}, 25-29 \mathrm{~kg} / \mathrm{m}^{2}$, and $\geq 30 \mathrm{~kg} / \mathrm{m}^{2}[8]$.

This study received ethical approval from the Syrian International University for Science and Technology (SIUST). The data were analyzed using SPSS 16.0. The significant difference between proportions or means was tested using chi-square test $\left(\chi^{2}\right)$ and $t$-test, respectively, with $P$ value $\leq 0.05$ as the level of significance.

\section{Results}

Out of the 500 chosen students, only 496 (284 males and 212 females) participated, and hence the responding rate was $99.2 \%$. The prevalence of acne among the students was $34.7 \%$ (172/496). As shown in Table 1, there was a significantly higher rate of acne among male students than that among females (42.9\% versus $23.6 \%$ ); $\chi^{2}=23.48, P<0.0001$. The mean age of students with acne was significantly younger in males $(18.13 \pm 0.59)$ than in females $(19.04 \pm 1.10) ; t=6.8$, $P<0.0001$. The study also found a significant difference in acne location $\left(\chi^{2}=67.7, P<0.001\right)$ in which both males (96.7\%) and females (92\%) demonstrated the highest rate of acne on the face followed by the shoulders.
TABLE 1: The Sociodemographic factors associated with acne formation.

\begin{tabular}{|c|c|c|c|c|}
\hline \multirow[b]{2}{*}{ Characteristics } & \multicolumn{2}{|c|}{ Acne positive } & \multirow{2}{*}{$\chi^{2}$} & \multirow[b]{2}{*}{$P$ value } \\
\hline & $\begin{array}{l}\text { Male (\%) } \\
N=284\end{array}$ & $\begin{array}{c}\text { Female (\%) } \\
N=212\end{array}$ & & \\
\hline Sex & $122(42.9)$ & $50(23.6)$ & 23.48 & $<0.0001$ \\
\hline Mean age \pm S.D & $18.13 \pm 0.59$ & $19.04 \pm 1.106$ & $t=6.8$ & $<0.0001$ \\
\hline \multicolumn{5}{|l|}{ Location } \\
\hline Face & $118(96.7)$ & $46(92)$ & 67.7 & $<0.001$ \\
\hline Shoulders & $48(39.3)$ & $17(34)$ & & \\
\hline Others & $23(18.9)$ & $7(14.0)$ & & \\
\hline \multicolumn{5}{|c|}{ Living with family } \\
\hline Yes & $50(41)$ & $33(66)$ & 54.9 & $<0.0001^{*}$ \\
\hline No & $72(59)$ & $17(34)$ & 6.27 & $<0.05^{* *}$ \\
\hline \multicolumn{5}{|l|}{ Stress } \\
\hline Always & $58(47.5)$ & $26(52)$ & 27.88 & $<0.0001^{*}$ \\
\hline Absence & $23(18.9)$ & $10(20)$ & 19.64 & $<0.001^{* *}$ \\
\hline Occasional & $41(33.6)$ & $14(28)$ & & \\
\hline \multicolumn{5}{|l|}{ Washing face/day } \\
\hline $1-2$ & $47(38.5)$ & $19(38)$ & 8.1 & $<0.025^{*}$ \\
\hline $3-5$ & $47(38.5)$ & $20(40)$ & 11.21 & $<0.004^{* *}$ \\
\hline$>5$ & $28(23)$ & $11(22)$ & & \\
\hline \multicolumn{5}{|c|}{ Washing body/week } \\
\hline $1-2$ & $42(34.4)$ & $8(16)$ & 14.0 & $<0.001^{*}$ \\
\hline $3-5$ & $51(41.8)$ & $11(22)$ & 4.44 & $>0.05^{* *}$ \\
\hline$>5$ & $29(23.8)$ & $31(62)$ & & \\
\hline \multicolumn{5}{|l|}{$\mathrm{BMI}\left(\mathrm{Kg} / \mathrm{m}^{2}\right)$} \\
\hline$<20$ & $5(18.5)$ & $3(8.1)$ & 8.74 & $0.05^{*}$ \\
\hline $20-24$ & $55(36.6)$ & $29(35.8)$ & 22.6 & $<0.0001^{* *}$ \\
\hline $25-29$ & $29(47.5)$ & $7(10.5)$ & & \\
\hline$\geq 30$ & $6(60)$ & $3(60)$ & & \\
\hline \multicolumn{5}{|l|}{ Menstrual cycles } \\
\hline Yes & & 40 & & \\
\hline No & & 10 & & \\
\hline
\end{tabular}

${ }^{*}$ Male, ${ }^{* *}$ female.

Students who wash their faces more than five time per day showed significantly lower prevalence of acne in males (23\%, $\left.\chi^{2}=8, P<0.025\right)$ and females $\left(22 \%, \chi^{2}=11.21, P<\right.$ $0.004)$.

On the other hand, significantly lower rate of acne was only demonstrated among those males washing their body more than five times per week $\left(23.8 \%, \chi^{2}=14.0, P<\right.$ $0.001)$, while no significant association was found in females regarding body wash $\left(62 \%, \chi^{2}=4.44, P>0.05\right)$.

Our study gave an evidence that acne prevalence significantly increased steadily $(18.5 \%, 36.6 \%, 47.5 \%, 60 \%)$ and $(8.1 \%, 35.8 \%, 10.5 \%, 60 \%)$ with the increased body mass index for BMI < 20, 20-24, 25-29 and $>30 \mathrm{~kg} / \mathrm{m} 2 ;\left(\chi^{2}=8.74\right.$, $P=0.05)$ and $\left(\chi^{2}=22.6, P<0.0001\right)$ in both males and females respectively.

With respect to the relation between degree of stress and acne development, our study showed that the highest 
significant prevalence in both males $\left(47.5 \%, \chi^{2}=27.88, P<\right.$ 0.0001 ) and females $\left(52 \%, \chi^{2}=19.64, P<0.001\right)$ was among students who were always subjected to stress compared to those who were occasionally or not subjected to stress. Male students who were living away from their family demonstrated significantly higher rate of acne, $\chi^{2}=$ $54.9, P<0.0001$, but similar finding was not detected among females. Regarding female students, $80 \%$ reported acne formation appeared during their menstrual cycles.

\section{Discussion}

The good response rate $(99.2 \%)$ reflected the interest of the students in this health problem. The prevalence of acne in this study was lower than reported among university students in Central Saudi Arabia (56.2\%), Turkey (40.1\%) and also among Singaporean adolescents $(88 \%)[3,6,9]$. This discrepancy could be attributed to variation in genetics, diet, or social factors (e.g., smoking).

The significant association between acne formation and sex of students that we detected was inconsistent with a study in Saudi [6]. However, similar finding was reported among school-aged adolescents in Malaysia, whereby acne was more common among males compared to females [10]. Males have higher prevalence of acne than females in our study and this conforms to studies in India, Dakar, Portugal, and Korea [1114]. One of the reasons why men having more acne lesion may be due to increased sebum production [14].

Males with acne that of mean age was significantly younger than females. This finding is consistent with several studies $[12,15,16]$. The result most probably is attributed to the male sex hormones (androgen) which increase during adolescence period. Androgen stimulates sebaceous glands to produce sebum, so hyperandrogenism is closely associated with acne formation [17].

Our significant association between acne occurrence and washing face daily in both sexes is in disagreement with several studies in which they stated that poor hygiene does not cause acne $[18,19]$. However, washing body frequently among males, but not in females, was found significantly associated with development of acne. There is a contradicting opinion about the association between acne formation and seasonal variation.

There is a conventional view that acne vulgaris exacerbates in winter, while South India study found acne prevalence is more in summer [12]. However, our study was conducted only during winter season in which, during cold weather, there is decrease in frequency of bathing among adolescents particularly male. That is why we observed significantly higher rate of acne among males with low frequency of bathing.

Degree of stress and its role with acne formation were stated by many authors $[5,14,20]$. They reported that emotional stress acts as an aggravating risk factor for development of acne. Moreover, Law et al., 2009, from his study in Hong Kong, found that higher perceived stress was positive predictor of acne among university students [21]. We confirmed the association between acne prevalence and degree of stress in which we found that students who always were subjected to continuous stress demonstrated higher rate of acne. Most probably, this relation could be attributed to the increased production of cortisol during emotional stress which in turn increased the sebum production [22,23].

Male students living within their families have significantly lower prevalence of acne. This could be related to higher dependency of male students on their parents for providing better personal hygiene and nutrition compared to their female counterparts. In addition, less psychological stress could not be excluded. Menstruation and its association with the increase of acne were detected in $80 \%$ of our female students which is consistent with several studies that stated that hyperandrogenicity during the menstrual cycle may have contributed to the acne formation [16, 24]. With regard to acne location, face is the commonest site for acne and this conforms to several authors [11-13, 15]. The relation between acne formation and BMI has been studied by many authors $[4,17,25,26]$. They found that acne was more prevalent among obese persons in women. We detected those obese students of both sexes to have higher acne rate.

Several explanations could explain the reasons and few of them are the excess androgens caused by obesity and psychological stress that could have resulted from being overweight which in turn cause surge of stress hormones that will worsen the acne $[17,25]$. In addition, diet may play a role [5]. However, we did not explore the diet factors related to acne in our study.

Our study has several limitations. We did not assess the severity of acne or referred students with bad acne for further management. In addition, the questions on stress were a subjective assessment. A proper tool that measures the degree of stress should be taken into account. Furthermore, statistical analysis to identify the unconfounding factors was not applied.

\section{Conclusion}

Acne is a health and psychological problem among university students which is highly related to age of youth particularly among males. There were several significant factors associated with acne formation in the study which were gender, BMI, and stress. Locality of acne and frequency of washing were also significantly associated with acne formation.

\section{Conflict of Interests}

The authors declare that there is no conflict of interests regarding the publication of this paper.

\section{Acknowledgments}

The authors thank the president of the Syrian International University for Science and Technology (SIUST) and the Scientific Research Committee for granting them the approval and assistance in conducting the study. 


\section{References}

[1] Y. Hussain, "Acne vulgaris in Nigerian adolescents-prevalence, severity, beliefs, perceptions, and practices," International Journal of Dermatology, vol. 48, no. 5, pp. 498-505, 2009.

[2] B. A. Yentzer, J. Hick, E. L. Reese, A. Uhas, S. R. Feldman, and R. Balkrishnan, "Acne vulgaris in the united states: a descriptive epidemiology," Cutis, vol. 86, no. 2, pp. 94-99, 2010.

[3] I. Ertam, Y. Babur, I. Unal, and S. Alper, "The frequency of skin diseases among students in a university clinic," Skinmed, vol. 8, no. 5, pp. 261-263, 2010.

[4] K. O. Abulnaja, "Changes in the hormone and lipid profile of obese adolescent Saudi females with acne vulgaris," Brazilian Journal of Medical and Biological Research, vol. 42, no. 6, pp. 501-505, 2009.

[5] Z. El-Akawi, N. A.-L. Nemr, K. Abdul-Razzak, and M. AlAboosi, "Factors believed by jordanian acne patients to affect their acne condition," Eastern Mediterranean Health Journal, vol. 12, no. 6, pp. 840-846, 2006.

[6] A. A. Al Robaee, "Prevalence, knowledge, beliefs and psychosocial impact of acne in university students in central Saudi Arabia," Saudi Medical Journal, vol. 26, no. 12, pp. 1958-1961, 2005.

[7] L. K. Dunn, J. L. O’Neill, and S. R. Feldman, "Acne in adolescents: quality of life, self-esteem, mood, and psychological disorders," Dermatology Online Journal, vol. 17, no. 1, 2011.

[8] Global Database on Body Mass Index, World Health Organization, Geneva, Switzerland, 2006, http://apps.who.int/bmi/ index.jsp?introPage=intro_3.html.

[9] H.-H. Tan, A. W. Tan, T. Barkham, X.-Y. Yan, and M. Zhu, "Community-based study of acne vulgaris in adolescents in Singapore," British Journal of Dermatology, vol. 157, no. 3, pp. 547-551, 2007.

[10] A. Hanisah, K. Omar, and S. A. Shah, "Prevalence of acne and its impact on the quality of life in school-aged adolescents in Malaysia," Journal of Primary Health Care, vol. 1, no. 1, pp. 20 25, 2009.

[11] A. Kane, S. O. Niang, A. C. Diagne, F. Ly, and B. Ndiaye, "Epidemiologic, clinical, and therapeutic features of acnein Dakar, Senegal," International Journal of Dermatology, vol. 46, 1, pp. S36-S38, 2007.

[12] B. Adityan and D. M. Thappa, "Profile of acne vulgarisa hospital-based study from South India," Indian Journal of Dermatology, Venereology and Leprology, vol. 75, no. 3, pp. 272278, 2009.

[13] J. M. Amado, M. E. Matos, A. M. Abreu et al., "The prevalence of acne in the north of Portugal," Journal of the European Academy of Dermatology and Venereology, vol. 20, no. 10, pp. 1287-1295, 2006.

[14] D. H. Suh, J. W. J. Shin, S. U. Min et al., “Treatment-seeking behaviors and related epidemiological features in Korean acne patients," Journal of Korean Medical Science, vol. 23, no. 6, pp. 969-974, 2008.

[15] J. Hassan, S. Grogan, D. Clark-Carter, H. Richards, and V. M. Yates, "The individual health burden of acne: appearancerelated distress in male and female adolescents and adults with back, chest and facial acne," Journal of Health Psychology, vol. 14, no. 8, pp. 1105-1118, 2009.

[16] S. Purdy, J. Langston, and L. Tait, "Presentation and management of acne in primary care: a retrospective cohort study," British Journal of General Practice, vol. 53, no. 492, pp. 525-529, 2003.
[17] M.-C. Tsai, W. Chen, Y.-W. Cheng, C.-Y. Wang, G.-Y. Chen, and T.-J. Hsu, "Higher body mass index is a significant risk factor for acne formation in schoolchildren," European Journal of Dermatology, vol. 16, no. 3, pp. 251-253, 2006.

[18] Mayo Clinic, Acne: causes, http://www.mayoclinic.com/health/ acne/DS00169/DSECTION=causes.

[19] U.S. Food and Drug Administration, Facing Facts about Acne, Consumer Health Information. Department of Health and Human Services, http://www.fda.gov/Forconsumers/consumerUpdates/ucm174521.htm.

[20] N. Hunger and C. Kumar, "A clinico-epidemiological study of adult acne: is it different from adolescent acne?" Indian Journal of Dermatology, Venereology and Leprology, vol. 78, pp. 335-341, 2012.

[21] M. P. M. Law, A. A. T. Chuh, A. Lee, and N. Molinari, "Acne prevalence and beyond: acne disability and its predictive factors among chinese late adolescents in Hong Kong," Clinical and Experimental Dermatology, vol. 35, no. 1, pp. 16-21, 2009.

[22] C. W. Choi, J. W. Choi, K. C. Park, and S. W. Youn, "Facial sebum affects the development of acne, especially the distribution of inflammatory acne," Journal of the European Academy of Dermatology and Venereology, vol. 27, no. 3, pp. 301-306, 2013.

[23] G. Yosipovitch, M. Tang, A. G. Dawn et al., "Study of psychological stress, sebum production and acne vulgaris in adolescents," Acta Dermato-Venereologica, vol. 87, no. 2, pp. 135-139, 2007.

[24] C. N. Collier, J. C. Harper, W. C. Cantrell, W. Wang, K. W. Foster, and B. E. Elewski, "The prevalence of acne in adults 20 years and older," Journal of the American Academy of Dermatology, vol. 58, no. 1, pp. 56-59, 2008.

[25] A. O'Connor, Really? The claim: excess weight raises the risk of acne, http://well.blogs.nytimes.com/2012/01/23/reallythe-claim-excess-weight-raises-the-risk-of-acne/?_r=1.

[26] J. A. Halvorsen, R. A. Vleugels, E. Bjertness, and L. Lien, "A population-based study of acne and body mass index in adolescents," Archives of Dermatology, vol. 148, no. 1, pp. 131-132, 2012. 


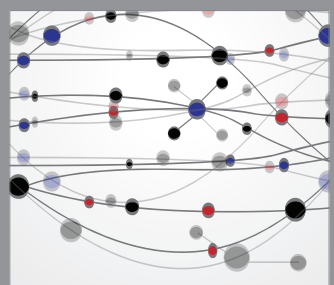

The Scientific World Journal
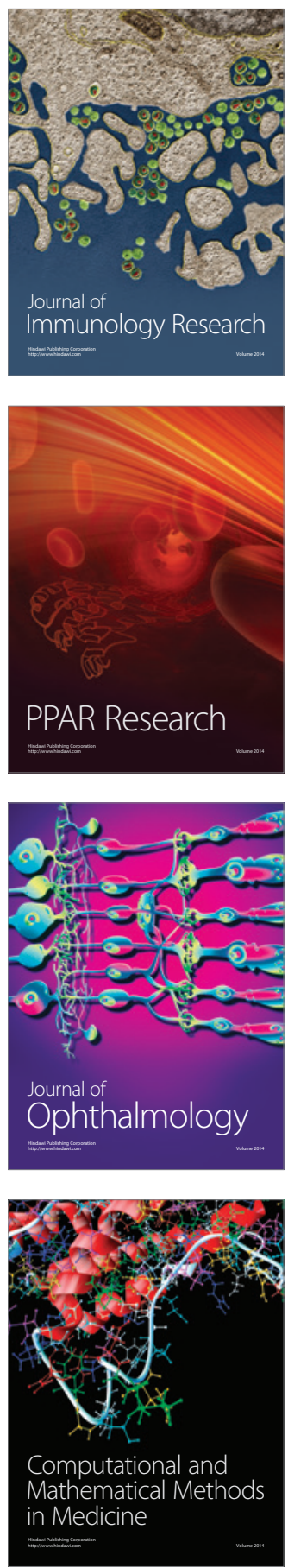

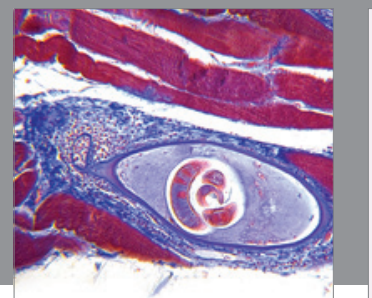

Gastroenterology

Research and Practice
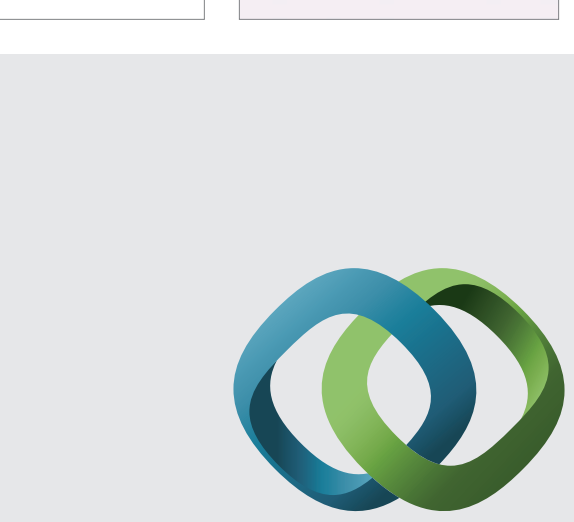

\section{Hindawi}

Submit your manuscripts at

http://www.hindawi.com
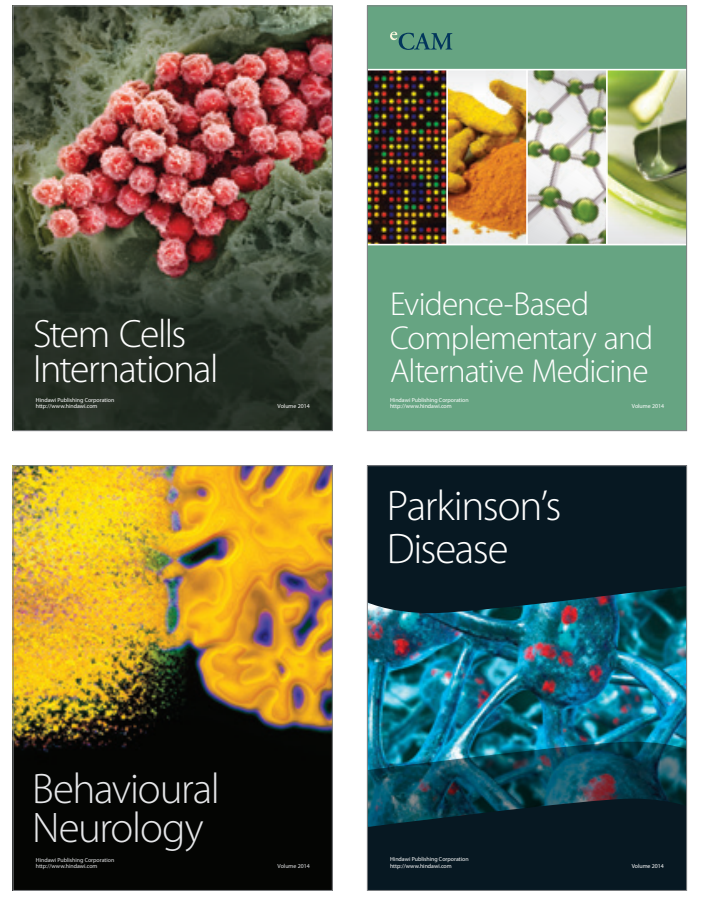
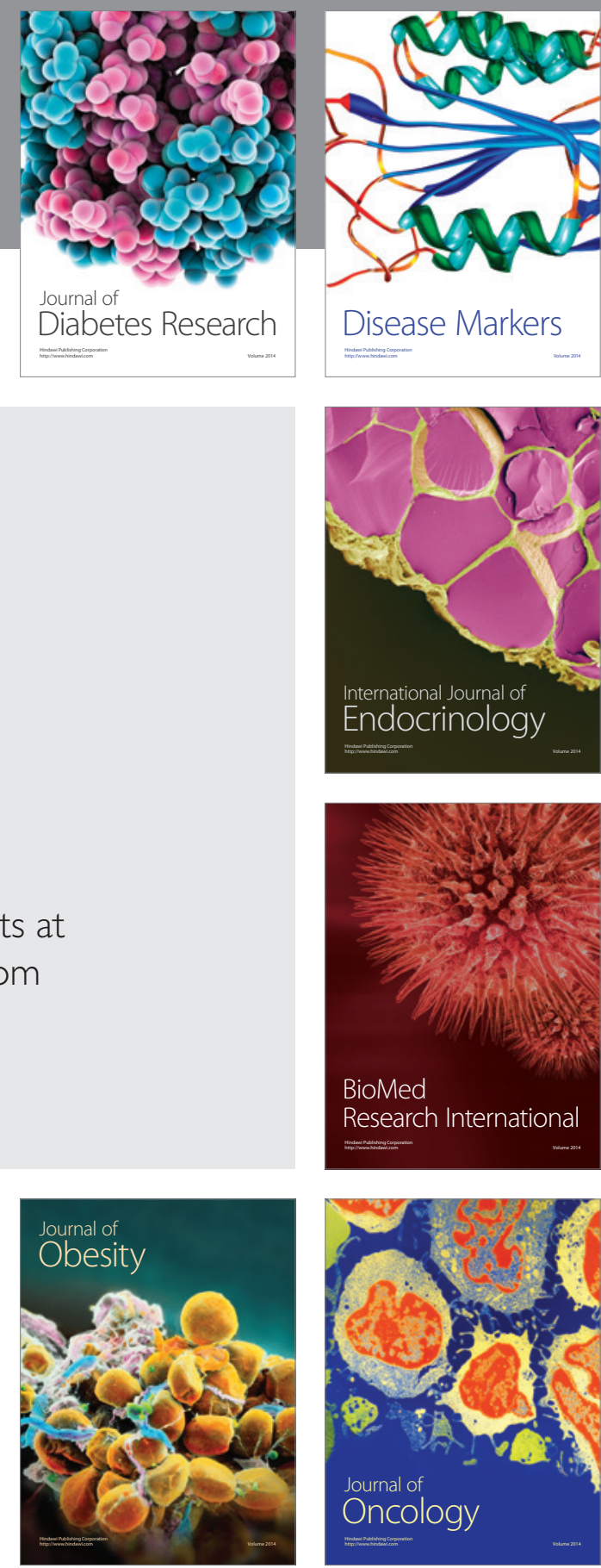

Disease Markers
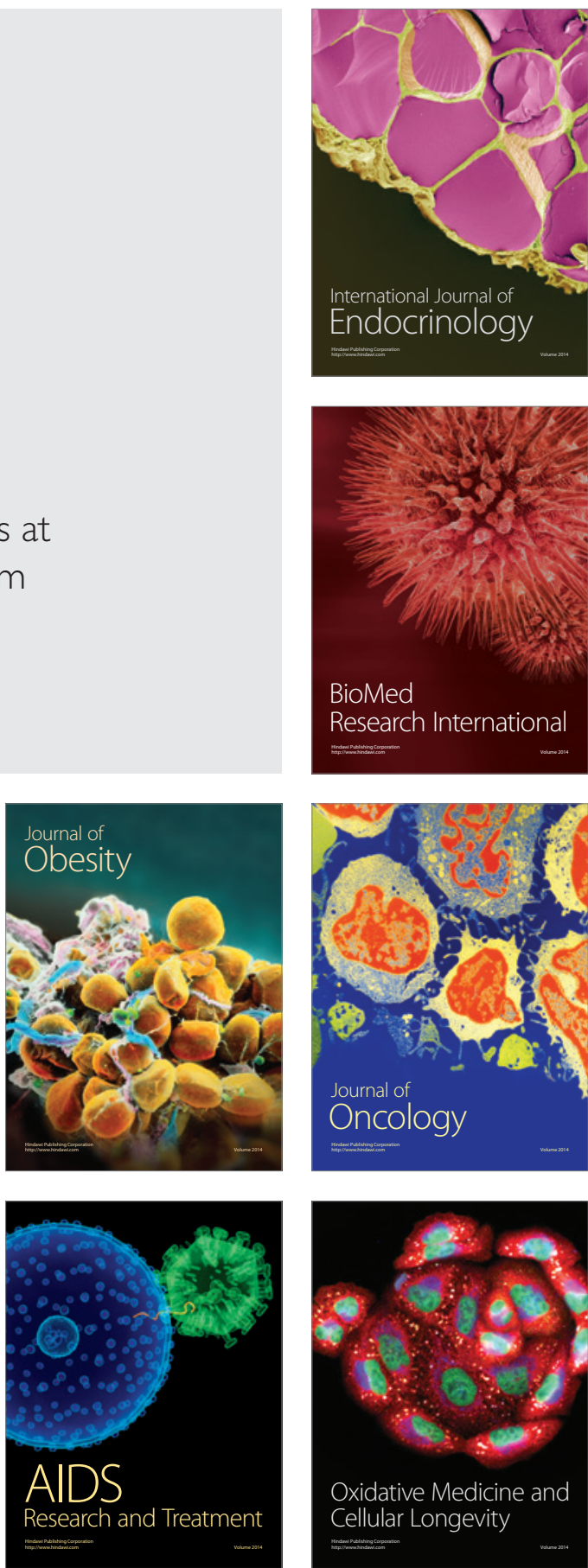